\title{
Deterministic Parallel Global Parameter Estimation for a Model of the Budding Yeast Cell Cycle
}

\author{
by
}

Thomas D. Panning*, Layne T. Watson ${ }^{+}$, Nicholas A. Allen*, Katherine C. Chen ${ }^{\dagger}$, Clifford A. Shaffer*, John J. Tyson ${ }^{\dagger}$

January 9, 2006

* Department of Computer Science

${ }^{+}$Departments of Computer Science and Mathematics

$\dagger$ Department of Biological Sciences

Virginia Polytechnic Institute and State University

Blacksburg, VA 24061

Running title: Deterministic Parallel Global Parameter Estimation 
Abstract. Two parallel deterministic direct search algorithms are used to find improved parameters for a system of differential equations designed to simulate the cell cycle of budding yeast. Comparing the model simulation results to experimental data is difficult because most of the experimental data is qualitative rather than quantitative. An algorithm to convert simulation results to mutant phenotypes is presented. Vectors of parameters defining the differential equation model are rated by a discontinuous objective function. Parallel results on a 2200 processor supercomputer are presented for a global optimization algorithm, DIRECT, a local optimization algorithm, MADS, and a hybrid of the two.

Key words: DIRECT (DIviding RECTangles) algorithm, Direct search, MADS (Mesh Adaptive Direct Search) algorithm, Computational biology

\section{Introduction}

Molecular cell biology describes how cells convert genes into behavior. This description includes how a cell creates proteins from genes, how those proteins interact, and how networks of interacting proteins determine physiological characteristics of the cell. The central biological question addressed here is how protein interactions regulate the cell cycle of budding yeast (Saccharomyces cerevisiae).

The budding yeast cell cycle consists of four phases (G1, S, G2, M), with cell division occurring in the final phase. A newborn cell starts in G1 phase (unreplicated DNA), during which time it grows to a sufficiently large size to warrant a new round of DNA synthesis (S phase). After DNA synthesis has completed, the cell passes briefly through G2 phase (replicated DNA) and then enters M phase (mitosis, where the two copies of each DNA molecule are separated and the cell divides, creating two new cells that are in G1 phase).

The protein interactions that govern these cell cycle events are modeled using differential equations that describe the rate at which each protein concentration changes. In general, the concentration of protein $\mathrm{A}$, written as $[\mathrm{A}]$, changes according to the differential equation

$$
\frac{d[\mathrm{~A}]}{d t}=\text { synthesis }- \text { degradation }- \text { binding }+ \text { dissociation }- \text { inactivation }+ \text { activation, }
$$

where "synthesis" is the rate at which new protein A molecules are synthesized from amino acids (which depends on the concentration of active messenger RNA molecules for a particular protein), "degradation" is the rate at which protein A is broken down into amino acids and polypeptide fragments (which depends on the activity of specific proteolytic enzymes), "binding" is the rate at 
which protein A combines with other molecules to form distinct molecular complexes, "dissociation" is the rate at which these complexes break apart, "inactivation" is the rate at which certain posttranslational modifications (e.g., phosphorylation) of protein A are made, and "activation" is the rate at which these modifications are reversed (e.g., dephosphorylation). Each of these rates is itself a function of the concentrations of the interacting species in the network. For example,

$$
\begin{aligned}
\text { synthesis } & =k_{1}[\text { transcription factor }] \\
\text { degredation } & =k_{2}[\text { proteolytic enzyme }][\mathrm{A}] \\
\text { binding } & =k_{3}[\mathrm{~A}][\mathrm{B}], \text { where } \mathrm{B} \text { is a binding partner, } \\
\text { dissociation } & =k_{4}[\mathrm{AB}], \\
\text { inactivation } & =\frac{k_{5}[\text { kinase }][\mathrm{A}]}{J_{5}+[A]}, \\
\text { activation } & =\frac{k_{6}[\text { phosphatase }]\left[\mathrm{A}_{p}\right]}{J_{6}+\left[\mathrm{A}_{p}\right]}, \text { where } \mathrm{A}_{p} \text { is the phosphorylated form of } \mathrm{A} .
\end{aligned}
$$

In these rate laws, the $k \mathrm{~s}$ are rate constants and the $J_{\mathrm{S}}$ are Michaelis constants. Other differential equations must be used to determine the temporal dynamics of the concentrations of the "transcription factor," "proteolytic enzyme," "kinase," etc.

The budding yeast cell cycle model consists of 36 such differential equations for two classes of variables: regulatory proteins and physiological "flags." The regulatory proteins are triggers for specific events of the budding yeast cell cycle: Cln2 triggers budding, Clb5 triggers DNA synthesis, Clb2 drives cells into mitosis, and Esp1 drives cells out of mitosis and back to G1. The physiological "flags" are dummy variables that track the strength of these trigger proteins. For example, "BUD" is an integral of the activity of Cln2; when BUD $=1$, a new bud is initiated. "ORI," an integral of [Clb5], represents the state of "origins of replication." When ORI = 1 ("fired" origins), DNA synthesis is initiated; at cell division, when $[\mathrm{Clb} 2]+[\mathrm{Clb} 5]$ drops below a threshold level, ORI is reset to zero ("licensed" origins). Finally, "SPN" represents the alignment of replicated chromosomes on the mitotic spindle. SPN is driven by Clb2 activity; i.e., SPN is an integral of [Clb2].

In the budding yeast model there are 143 rate constant parameters $\left(k \mathrm{~s}, J_{\mathrm{s}}\right.$, etc. $)$. In some cases, these parameters can be calculated directly from laboratory experiments (e.g., apparent protein half-lives), but most parameters are difficult to obtain directly from experimentation. Normally, modelers determine the remaining parameters by making educated guesses, solving the differential equations numerically, comparing the simulation results with laboratory data, and then refining their guesses. (Modelers call this process "parameter twiddling" [1].) For the budding yeast cell 
cycle, the laboratory data consists of observed phenotypes of more than 100 mutant yeast strains constructed by disabling and/or over-expressing the genes that encode the proteins of the regulatory network.

Although parameter twiddling is extremely tedious, it was used to obtain a parameter vector $\left(s_{1}, s_{2}, \ldots, s_{143}\right)$ for which the model's predictions are consistent with almost all of the budding yeast mutants being modeled. Obviously, the modelers would prefer a method that allows them to spend more time working on the model and less time twiddling parameters. In addition, a person can only keep track of a few parameters at one time, which makes it easy for him or her to unwittingly miss a portion of the parameter space. For these reasons, modelers would prefer to use a tool that determines "good" parameters automatically, quickly and accurately.

Section 3 describes a proposed mathematical formulation of "good" that allows a computer code to find an acceptable vector of parameters. This formulation uses a discontinuous objective function that evaluates to zero when there is a perfect match between the experimental data (mutant phenotypes) and the simulation results, and it evaluates to increasingly larger numbers to indicate worse matches. Another possible formulation for future consideration would use a smooth system of inequalities that would be satisfied if and only if the simulation results are acceptable.

Section 2 describes the biological problem in some detail. Section 3 formulates a discontinuous objective function, reflecting biological criteria for an acceptable model. Two deterministic algorithms, DIRECT and MADS, that are applicable to global parameter estimation, are briefly described in Section 4. Numerical results on a parallel supercomputer (2200 processor System X) are given in Section 5. Parallel efficiency and scalability are important issues to be addressed separately - the emphasis here is on the biological problem, the discontinuous objective function formulation, and the practical applicability of DIRECT and MADS to such optimization problems.

Throughout this paper, the observed phenotype refers to the phenotype that was recorded in a laboratory experiment. The predicted phenotype refers to the phenotype that the mathematical model (with its associated parameters) predicts. The wild type is the normal strain of an organism. The mutant strains have genetic changes that make them behave differently from the wild type in some way. 


\section{Observed and Predicted Phenotypes}

Experimental biologists have studied many budding yeast mutants to learn about the cell cycle regulatory system. Of these mutants, 115 were chosen to model (see Appendix A). A model of budding yeast can be considered acceptable only if it is able to duplicate the behavior of most of these mutants. (It would be too much to expect a model to account for all the "observations" because of lingering uncertainties about the reaction network and inevitable mistakes in phenotyping mutants.) When the model is used to simulate a mutant, the parameter vector can be changed only in ways that are dictated by the genetic changes in the mutant. Consider the hypothetical proteins A and B presented in the previous section: if a mutant had a modified form of B that did not bind to $\mathrm{A}$, then in the parameter vector for that mutant, $k_{3}$ would be set to zero and all the other parameters would be kept at the wild type values.

When comparing the model to the experimental data, it is important to realize that much of the data from laboratory experiments is qualitative. Such data is of the form "the cell is viable but considerably larger than wild type cells" or "the cell arrests in G1 phase and eventually dies." The quantitative data that is available (e.g., duration of G1 phase, cell mass at division) is generally imprecise. With all these uncertainties, there may be many, clustered parameter vectors that allow the model to reproduce the experimental data sufficiently well.

\subsection{RULES OF VIABILITY}

To compare solutions of the differential equations with experimental data, it is necessary to predict cell cycle properties from a simulation of regulatory protein dynamics. Viability is determined by four rules:

1. The simulation must execute the following events in order, or else the modeled cell is considered inviable:

(a) origin relicensing (modeled by a drop in [Clb2] $+[\mathrm{Clb} 5]$ below a threshold $K_{\mathrm{ez} 2}$ );

(b) origin activation (due to a subsequent rise in [Clb2] and [Clb5], causing [ORI] to increase above one) before a wild-type cell in the same medium would divide twice;

(c) spindle alignment (due to a rise in [Clb2], causing [SPN] to increase above one);

(d) Esp1 activation (modeled by [Esp1] increasing above 0.1);

(e) cellular division (modeled by [Clb2] dropping below a threshold $K_{e z}$ ).

2. The cell is inviable if division occurs in an "unbudded cell" (i.e., if [BUD] does not reach the value 0.8 before event (e) occurs). 


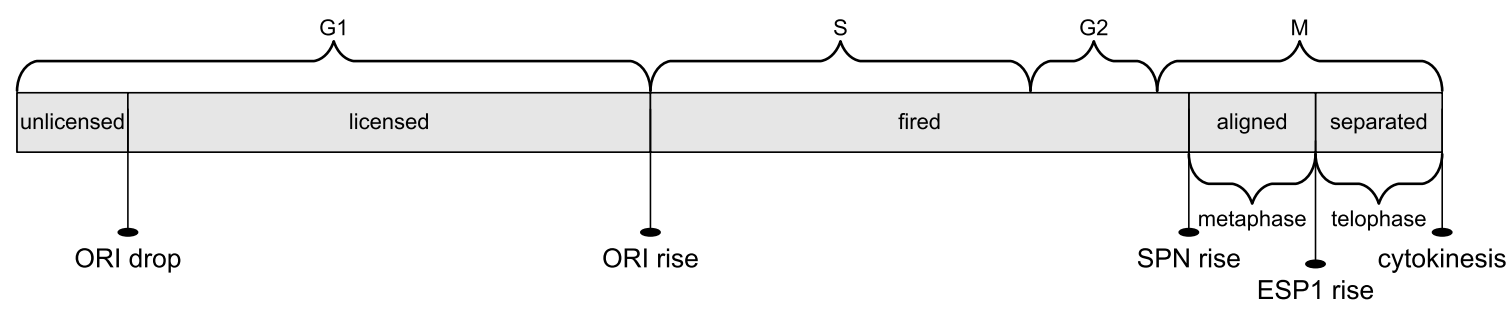

Figure 1. The five stages of the cell cycle, delineated by the events described in the first rule of viability. The four biological phases of the cell are above the stages, and two of the phases within $\mathrm{M}$ phase are shown below their corresponding stages.

3. The cell cycle should be stable, i.e., the squared relative differences of the masses and G1 phase durations in the last two cycles should both be less than 0.05 .

4. Lastly, the modeled cell is considered inviable if cell mass at division is greater than four times or less than one-fourth times the steady-state mass at division of the wild type in the same medium.

As mentioned in Section 1, the physiological flags are reset when certain events (a-e) occur. For a complete description of the resetting rules, see [5].

The viability rules are used by an algorithm[2] (called a transform) that outputs a phenotype from a solution of the differential equations. The transform keeps track of what stage the cell is in, where the stages are demarcated by the events (a-e) above. The first stage is unlicensed, which ends when the first event, origin relicensing, occurs. The other four stages are, in chronological order, licensed, fired, aligned, and separated. When the simulated cell is in the separated stage, cellular division signals the transition back to the unlicensed stage. The relations among the stages, events, and biological phases of the cell are shown in Figure 1. If one of the rules of viability is broken, the transform sets an error flag and records the stage when the error occurred and the number of cycles (i.e., cell divisions) completed from the time when the mutation was expressed to the time when the cell arrested.

\subsection{INITIAL CONDITIONS}

In the experimental data set, many of the mutations are conditional, that is, the mutant cells when grown under "normal" conditions (say, glucose medium at room temperature) behave like wild-type cells, but when grown under "restrictive" conditions (say, galactose medium or elevated temperature) the cells express the genetic mutation and the aberrant phenotype. To model this situation at sample points in parameter space, start a "wild-type" simulation from arbitrary (but reasonable) initial conditions and integrate the differential equations for two full cycles, in order 
to wash out any effects of the initial conditions. Then record the state of the control system just after origin relicensing (see Figure 1) at the beginning of the third cycle. These recorded values are used as initial conditions for simulating a steady state wild-type cell and for simulating each of the mutants.

\section{Formulation as a Discontinuous Minimization Problem}

The objective function takes the observed phenotype and predicted phenotype for all of the mutants and computes a nonnegative score. Zero indicates a perfect match and larger numbers indicate increasingly worse matches. The ensuing discussion uses the symbol $O$ for observed phenotype values and $P$ for predicted phenotype values.

A budding yeast phenotype for a single mutant is represented by a six-tuple $(v, g, m, a, t$, c), where the viability $v \in\{$ viable, inviable\}, the real number $g>0$ is the steady state length of the G1 phase in minutes, the real number $m>0$ is the steady state mass at division expressed as a multiple of the wild type's steady state mass at division in the same medium (e.g., glucose or galactose), the stage when arrest occurs is

$$
a \in A=\{\text { unlicensed, licensed, fired, aligned, separated }\}
$$

the positive integer $t$ is the arrest type (e.g., if events occur in improper order), and the nonnegative integer $c$ is the number of successful cycles completed. The observed and predicted phenotypes are written $O=\left(O_{v}, O_{g}, O_{m}, O_{a}, O_{t}, O_{c}\right)$ and $P=\left(P_{v}, P_{g}, P_{m}, P_{a}, P_{t}, P_{c}\right)$, respectively. Arrest types cannot be compared unless the stage of arrest is the same for both phenotypes.

The rating function, $R$, compares the observed and predicted phenotypes for a mutant. This rating function is a modified version of the one developed by N. Allen et al. [2]; the only difference is that if $P_{v}$ is missing (if integration fails for some reason), then $R(O, P)=\omega_{v}$. The rating function is split into four cases depending on the viability of the observed and predicted phenotypes. If $O_{v}=$ inviable, $P_{v}=$ viable, and $O_{c}$ is missing, then $R(O, P)=\omega_{v}$, the same as if $O_{c}=0$. Otherwise, if a needed classifier is missing, the term is simply dropped and does not contribute to the objective function. In the case that classifiers are missing, this allows the objective function value to be at or near zero when viability is in agreement between the phenotypes, and forces larger objective function values when viability is not in agreement. 
In what follows, the $\omega$ s and $\sigma$ s are constants defined in Table 1. The rating function $R(O, P)$ when all classifiers are present is given by

$$
\omega_{g} \times\left(\frac{O_{g}-P_{g}}{\sigma_{g}}\right)^{2}+\omega_{m} \times\left(\frac{\ln \frac{O_{m}}{P_{m}}}{\sigma_{m}}\right)^{2},
$$

if $O_{v}=$ viable and $P_{v}=$ viable, by

$$
\omega_{v} \times \frac{1}{1+P_{c}}
$$

if $O_{v}=$ viable and $P_{v}=$ inviable, by

$$
\delta_{O, P}+\omega_{c} \times\left(\frac{O_{c}-P_{c}}{\sigma_{c}}\right)^{2}
$$

if $O_{v}=$ inviable and $P_{v}=$ inviable, and by

$$
\omega_{v} \times \frac{1}{1+O_{c}}
$$

if $O_{v}=$ inviable and $P_{v}=$ viable. $\delta$ is a real-valued discrete function used to assess a penalty for the arrest stage and type, given by

$$
\delta_{O, P}= \begin{cases}\omega_{a}, & \text { if } O_{a} \neq P_{a} \\ \omega_{t}, & \text { if } O_{a}=P_{a} \text { and } O_{t} \neq P_{t} \\ 0, & \text { if } O_{a}=P_{a} \text { and } O_{t}=P_{t}\end{cases}
$$

The rating function is tuned by parameters that allow adjusting the relative importance of classifiers. The parameters given by Table 1 were set so that a rating of around ten indicates a critical error in the model's prediction of a phenotype.

\begin{tabular}{clr} 
Symbol & Definition & Value \\
\hline$\omega_{g}$ & G1 length weight & 1.0 \\
$\sigma_{g}$ & G1 length scale & 10.0 \\
$\omega_{m}$ & Mass at division weight & 1.0 \\
$\sigma_{m}$ & Mass at division scale & $\ln 2$ \\
$\omega_{a}$ & Arrest stage weight & 10.0 \\
$\omega_{t}$ & Arrest type weight & 5.0 \\
$\omega_{c}$ & Cycle count weight & 10.0 \\
$\sigma_{c}$ & Cycle count scale & 1.0 \\
$\omega_{v}$ & Viability weight & 40.0
\end{tabular}

Table 1. Constants used in the objective function. 
Denote the real numbers by $\mathcal{R}$, the nonnegative integers $\{0,1,2, \ldots\}$ by $\mathcal{Z}_{+}$, and the integers by $\mathcal{Z}$. Let

$$
\mathcal{P}=\{(v, g, m, a, t, c)\}=\{\text { viable, inviable }\} \times(0, \infty)^{2} \times A \times\{1, \ldots, 10\} \times \mathcal{Z}_{+}
$$

be the space of all budding yeast phenotypes and let the domain of the objective function be the box

$$
\Omega=\left\{x \in \mathcal{R}^{143}: \ln \left(s_{i} / u_{i}\right) \leq x_{i} \leq \ln \left(s_{i} \times u_{i}\right), i=1, \ldots, 143\right\}
$$

where $u \in \mathcal{R}^{143}$ is a vector of positive scale factors (most components of $u$ are approximately 100) reflecting a priori limits on the rate constants, and $s \in \mathcal{R}^{143}$ is the modeler's best guess point. Let $T_{j}: \Omega \rightarrow \mathcal{P}$ define the simulated phenotype of the $j$ th mutant with the parameters $\left(p_{1}, \ldots, p_{143}\right)=$ $\left(e^{x_{1}}, \ldots, e^{x_{143}}\right)$, where $\left(x_{1}, \ldots, x_{143}\right) \in \Omega$. Then the objective function $f: \Omega \rightarrow[0, \infty)$ is defined by

$$
f(x)=\sum_{j=1}^{N_{m}} \mu_{j} R\left(O_{j}, T_{j}(x)\right),
$$

where $N_{m}$ is the number of mutants simulated, and $\mu_{i}>0$ indicates the relative importance of the $i$ th mutant. The objective function value at the best previously known point [5] is 470 .

\section{Algorithms}

This section describes two algorithms that show promise for optimizing the discontinuous objective function described in the previous section. Consider the problem of minimizing $f: B \rightarrow$ $\mathcal{R}$, where $B=[l, u] \subset \mathcal{R}^{n}$ is a box.

\subsection{DIRECT}

The DIRECT (Dividing Rectangles) global minimization algorithm [11] requires the objective function to be Lipschitz continuous to guarantee convergence. Even though the objective function used here is discontinuous, the DIRECT algorithm seems to be an efficient and reasonable deterministic sampling strategy worth trying.

The DIRECT algorithm is one of a class of deterministic direct search algorithms that does not require gradients. It works by iteratively dividing the search domain into boxes that have exactly one function value at the box's center. In each iteration, the algorithm determines which boxes are most likely to contain a better point than the current minimum point- these boxes are called "potentially optimal". It then subdivides the potentially optimal boxes along their 
longest dimensions. Intuitively, a box is considered potentially optimal if it has the potentially best function value for a given Lipschitz constant. The formal definition from [11] follows.

DEFINITION 1. Suppose that the unit hypercube has been partitioned into $m$ (hyper) boxes. Let $c_{i}$ denote the center point of the $i$ th box, and let $d_{i}$ denote the distance from the center point to the vertices. Let $\epsilon \geq 0$ be a positive constant. A box $j$ is said to be potentially optimal if there exists some $\tilde{K}>0$ such that for all $i=1, \ldots, m$,

$$
\begin{aligned}
& f\left(c_{j}\right)-\tilde{K} d_{j} \leq f\left(c_{i}\right)-\tilde{K} d_{i}, \quad \text { for all } i=1, \ldots, m, \\
& f\left(c_{j}\right)-\tilde{K} d_{j} \leq f_{\min }-\epsilon\left|f_{\min }\right| .
\end{aligned}
$$

The DIRECT algorithm is described by the following six steps [10].

Step 1. Normalize the design space $B$ to be the unit hypercube. Sample the center point $c_{i}$ of this hypercube and evaluate $f\left(c_{i}\right)$. Initialize $f_{\min }=f\left(c_{i}\right)$, evaluation counter $m=1$, and iteration counter $t=0$.

Step 2. Identify the set $S$ of potentially optimal boxes.

Step 3. Select any box $j \in S$.

Step 4. Divide the box $j$ as follows:

(1) Identify the set $I$ of dimensions with the maximum side length. Let $\delta$ equal one-third of this maximum side length.

(2) Sample the function at the points $c \pm \delta e_{i}$ for all $i \in I$, where $c$ is the center of the box and $e_{i}$ is the $i$ th unit vector.

(3) Divide the box $j$ containing $c$ into thirds along the dimensions in $I$, starting with the dimension with the lowest value of $w_{i}=\min \left\{f\left(c+\delta e_{i}\right), f\left(c-\delta e_{i}\right)\right\}$, and continuing to the dimension with the highest $w_{i}$. Update $f_{\min }$ and $m$.

Step 5. Set $S=S-\{j\}$. If $S \neq 0$ go to Step 3 .

Step 6. Set $t=t+1$. If iteration limit or evaluation limit has been reached, stop. Otherwise, go to Step 2 . 


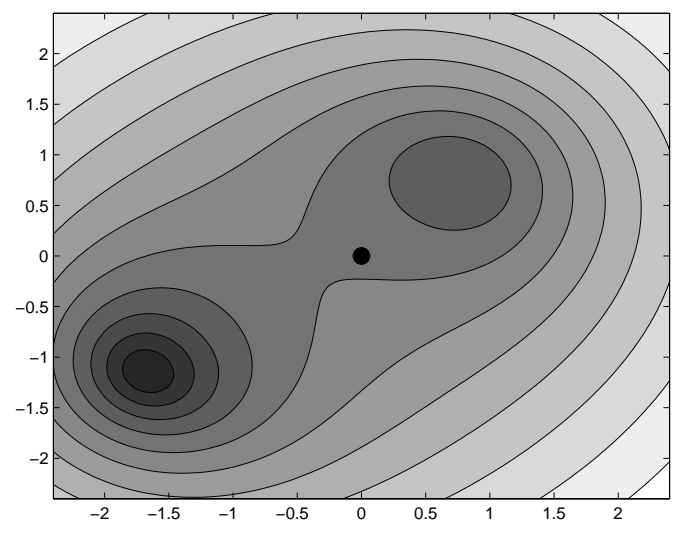

initial

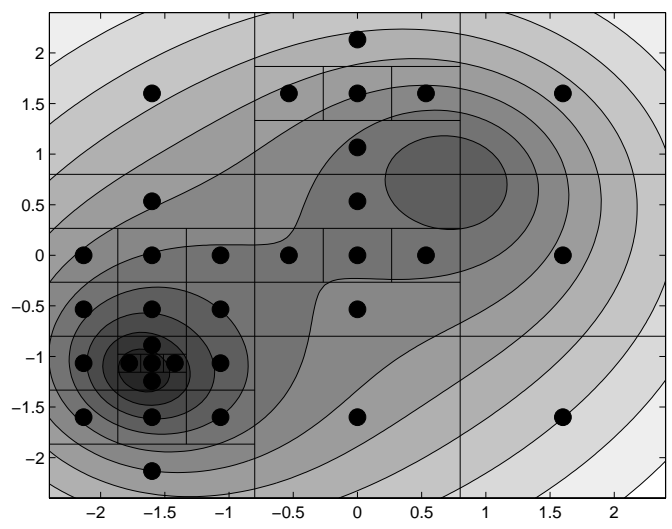

after 5 iterations

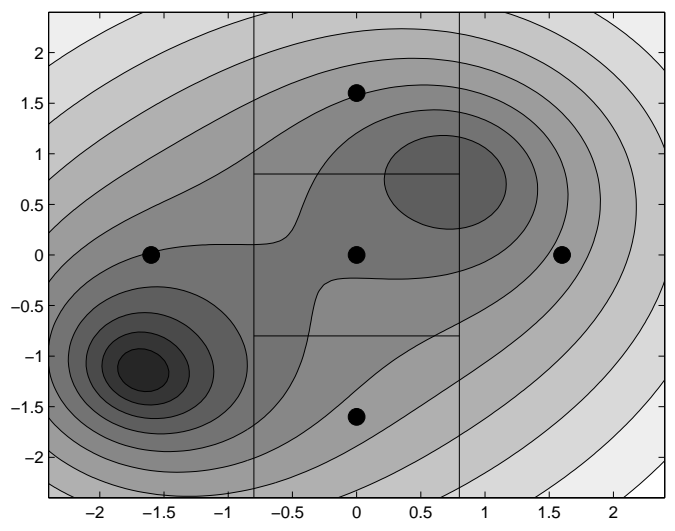

after 1 iteration

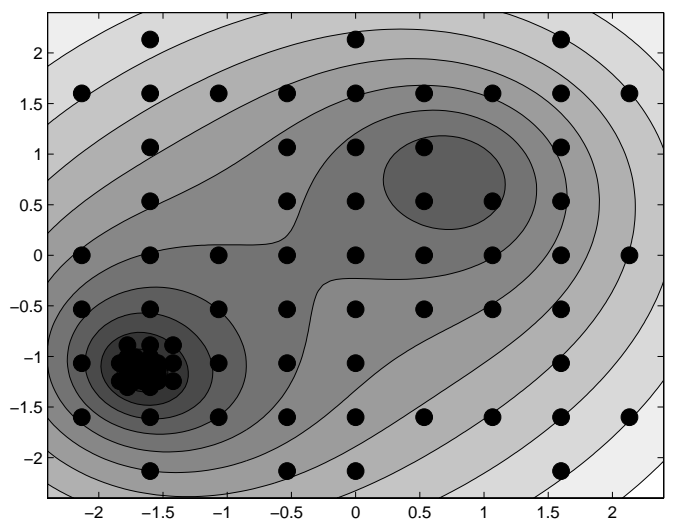

after 11 iterations

Figure 2. These graphs show the function evaluations that DIRECT performed after zero, one, five, and eleven iterations. Comparing the first and second graphs shows how DIRECT divides a two-dimensional box. The second and third graphs include the rectangles that DIRECT had created. After five iterations, DIRECT has found the global minimum at $(-1.8,-1.1)$. After the fifth iteration DIRECT has explored the domain, subdividing most of the larger boxes. After eleven iterations, DIRECT has evaluated the function at points near the local minimum.

For an illustration of how the DIRECT algorithm searches the domain on an example problem, see Figure 2. Both serial [10] and parallel [7] versions of DIRECT have been described in the literature.

\subsection{MADS}

A MADS (Mesh Adaptive Direct Search) algorithm, as defined by Audet and Dennis [4], minimizes a nonsmooth function $f: \mathcal{R}^{n} \rightarrow \mathcal{R} \cup\{+\infty\}$ under general constraints $x \in \Omega \subseteq \mathcal{R}^{n}$, $\Omega \neq \emptyset$. If $\Omega \neq \mathcal{R}^{n}$, the algorithm works with $f_{\Omega}$, which is equal to $f$ on $\Omega$ and $+\infty$ outside $\Omega$. Using $f_{\Omega}$ in lieu of $f$ is called a "barrier" approach to handling arbitrary constraints $x \in \Omega$. 
In each iteration, a MADS algorithm evaluates the objective function $f_{\Omega}$ at a finite number of trial points. Central to these algorithms is the concept of a mesh, which is a discrete set of points in $\mathcal{R}^{n}$. Every previous trial point must lie on the current mesh, and in each iteration the algorithm may only generate new trial points on the current mesh. This is not as restrictive as it might sound because the algorithm changes the mesh after each iteration (with the restriction that all previously evaluated points remain in the new mesh).

To further define the mesh, three entities $-\Delta_{k}^{m}, D, S_{k}$-must be introduced. First, the mesh size parameter $\Delta_{k}^{m}>0$ controls the granularity of the mesh at iteration $k$; after the $k$ th iteration, $\Delta_{k+1}^{m}$ is adjusted from $\Delta_{k}^{m}$ depending on the success of that iteration. The second entity is an $n \times n_{D}$ matrix $D$, where each column $D \cdot j=G z_{j}$ (for $j=1,2, \ldots, n_{D}$ ) for some fixed nonsingular generating matrix $G \in \mathcal{R}^{n \times n}$ and nonzero integer vector $z_{j} \in \mathcal{Z}^{n}$. The columns of $D$ must also be a positive spanning set, $\operatorname{Pos}(D)=\mathcal{R}^{n}$ (i.e., the cone generated by nonnegative combinations of columns of $D$ spans $\mathcal{R}^{n}$ ). Lastly, $S_{k}$ is the set of points where the objective function has been evaluated by the start of iteration $k$. Now that those entities have been introduced, the current mesh can be precisely defined.

DEFINITION 2. At iteration $k$, the current mesh is defined to be

$$
M_{k}=\bigcup_{x \in S_{k}}\left\{x+\Delta_{k}^{m} D z: z \in \mathcal{N}^{n_{D}}\right\}
$$

This definition ensures that all previously evaluated points are included in the mesh. It also shows that a smaller $\Delta_{k}^{m}$ will result in a more refined mesh, while a larger $\Delta_{k}^{m}$ will create a coarser mesh.

Now that the mesh has been defined, the iterations of a MADS algorithm can be described. Each iteration consists of two steps: the SEARCH step and the POLL step. The SEARCH step may evaluate $f_{\Omega}$ at any finite number of mesh points. At which mesh points $f_{\Omega}$ is evaluated depends on the precise MADS algorithm in use. A MADS algorithm may even do zero evaluations in the SEARCH step; the SEARCH step is said to be empty when no points are considered. If the SEARCH step fails to find a mesh point at which $f_{\Omega}$ is less than $\min _{x \in S_{k}} f_{\Omega}(x)$, then the algorithm performs the POLL step by generating and evaluating $f_{\Omega}$ at new trial points around the current incumbent solution $x_{k}$, where $f_{\Omega}\left(x_{k}\right)=\min _{x \in S_{k}} f_{\Omega}(x)$. The poll size parameter $\Delta_{k}^{p}$ limits the distance between $x_{k}$ and the new trial points. The set of new trial points is called a frame, and $x_{k}$ is called the frame center. The MADS frame is constructed using $x_{k}, \Delta_{k}^{p}, \Delta_{k}^{m}$, and $D$ to obtain a set $D_{k}$ of positive spanning directions. 
DEFINITION 3. At iteration $k$, the MADS frame is defined to be the set

$$
P_{k}=\left\{x_{k}+\Delta_{k}^{m} d: d \in D_{k}\right\} \subset M_{k}
$$

where $D_{k}$ is a positive spanning set such that $0 \notin D_{k}$ and for each $d \in D_{k}$,

- $d$ can be written as a nonnegative integer combination of the columns of $D: d=D u$ for some vector $u \in \mathcal{N}^{n_{D}}$,

- the distance from the frame center $x_{k}$ to a frame point $x_{k}+\Delta_{k}^{m} d \in P_{k}$ is bounded by a constant times the poll size parameter: $\Delta_{k}^{m}\|d\|_{\infty} \leq \Delta_{k}^{p}\|D\|_{\infty}$,

- limits (as defined in Coope and Price [6]) of the normalized sets $D_{k}$ are positive spanning sets.

The algorithm evaluates $f_{\Omega}$ at points in the frame $P_{k}$ until it encounters an improved point $x^{*}\left(f_{\Omega}\left(x^{*}\right)<f_{\Omega}\left(x_{k}\right)\right)$ or it has evaluated $f_{\Omega}$ at all of the points in $P_{k}$.

After the algorithm has executed the SEARCH step and (conditionally) the POLL step, it sets the mesh size and poll size parameters, $\Delta_{k+1}^{m}$ and $\Delta_{k+1}^{p}$, for the next iteration. If the iteration successfully found a better mesh point $x_{k+1}$ such that $f_{\Omega}\left(x_{k+1}\right)<f_{\Omega}\left(x_{k}\right)$, then $\Delta_{k+1}^{m}$ will be larger than or equal to $\Delta_{k}^{m}$; otherwise, $\Delta_{k+1}^{m}$ will be smaller than $\Delta_{k}^{m}$. The poll size parameter $\Delta_{k+1}^{p}$ must be set such that $\Delta_{k+1}^{m} \leq \Delta_{k+1}^{p}$, and it must satisfy

$$
\liminf _{k \rightarrow \infty} \Delta_{k}^{m}=0 \Longleftrightarrow \liminf _{k \rightarrow \infty} \Delta_{k}^{p}=0
$$

Exactly how $\Delta_{k+1}^{m}$ and $\Delta_{k+1}^{p}$ are generated is determined by the individual algorithm in use. A typical algorithm uses the following rules to set the mesh size parameter: $\Delta_{0}^{m}=1$, and

$$
\Delta_{k+1}^{m}= \begin{cases}\Delta_{k}^{m} / 4, & \text { if } x_{k} \text { is a minimizing } \\ & \text { frame center } \\ 4 \Delta_{k}^{m}, & \text { if an improved mesh } \\ & \text { point is found, and } \\ & \text { if } \Delta_{k}^{m} \leq \frac{1}{4} \\ \Delta_{k}^{m}, & \text { otherwise. }\end{cases}
$$

The same example algorithm then uses the simple rule $\Delta_{k}^{p}=\sqrt{\Delta_{k}^{m}}$ to determine the value of $\Delta_{k+1}^{p}$. These rules ensure that $\Delta_{k}^{m}$ is always a power of $1 / 4$ less than or equal to one, and $\Delta_{k}^{m}$ is always less than or equal to $\Delta_{k}^{p}$.

In summary, the MADS class of algorithms is described by the following five steps.

Step 1. Let $x_{0} \in \Omega$ and $0<\Delta_{0}^{m} \leq \Delta_{0}^{p}$. Let $D$ be an $n \times n_{D}$ matrix with the properties described earlier. Set the iteration counter $k:=0$. 
Step 2. Perform the SEARCH step. This step varies among the individual algorithms; in all algorithms $f_{\Omega}$ is evaluated at a finite subset of points (called trial points) on the mesh $M_{k}$. If a trial point $y$ is found such that $f_{\Omega}(y)<f_{\Omega}\left(x_{k}\right)$, then the algorithm may go to Step 4 with $x_{k+1}:=y$.

Step 3. Perform the POLL step, evaluating $f_{\Omega}$ at points from the frame $P_{k} \subset M_{k}$ until a frame point $x_{k+1}$ is found with $f_{\Omega}\left(x_{k+1}\right)<f_{\Omega}\left(x_{k}\right)$ or $f_{\Omega}$ has been evaluated at all of the points in $P_{k}$.

Step 4. Update $\Delta_{k+1}^{m}$ and $\Delta_{k+1}^{p}$ according to the specific algorithm's rules. In all algorithms,

(1) $\Delta_{k+1}^{m}$ is greater than or equal to $\Delta_{k}^{m}$ if an improved mesh point is found,

(2) $\Delta_{k+1}^{m}$ is less than $\Delta_{k}^{m}$ if an improved mesh point is not found,

(3) $\Delta_{k+1}^{p}$ is greater than or equal to $\Delta_{k+1}^{m}$, and

(4) $\liminf _{j \rightarrow \infty} \Delta_{j}^{m}=0$ if and only if $\liminf _{j \rightarrow \infty} \Delta_{j}^{p}=0$.

Step 5. If an appropriate stopping criterion has been met, stop. Otherwise, set $k:=k+1$ and go back to Step 2 .

The previous discussion presents the MADS class of algorithms. The following discussion describes a specific instance of the class, and Figure 3 shows how that algorithm behaves on an example problem. To emphasize the POLL step of the algorithm, there is no SEARCH step in the algorithm presented here.

In this MADS algorithm,

$$
D=\left(\begin{array}{cccc}
1 & 0 & -1 & 0 \\
0 & 1 & 0 & -2
\end{array}\right)
$$

Notice that a MADS mesh constructed using this matrix is identical to a mesh constructed using the matrix

$$
B=\left(\begin{array}{cccc}
1 & 0 & -1 & 0 \\
0 & 1 & 0 & -1
\end{array}\right)
$$

However, $\|D\|_{\infty}=2$ while $\|B\|_{\infty}=1$; thus, a MADS frame constructed using $D$ instead of $B$ will extend twice as far in every direction. From $D$, the matrix $D_{k}$ is generated (using random coefficients as described in [4]) at the beginning of the $k$ th iteration so that it is a positive spanning set, and so that the (normalized) columns of $D_{i}$, for $i=1,2, \ldots$, are dense in the unit circle $\mathcal{S}^{1}$.

The mesh size parameter $\Delta^{m}$ is updated according to the rules:

$$
\begin{aligned}
\Delta_{0}^{m} & =1, \\
\Delta_{k+1}^{m} & = \begin{cases}\Delta_{k}^{m} / 4, & \text { if } x_{k} \text { is a minimizing frame center, } \\
4 \Delta_{k}^{m}, & \text { if an improved mesh point is found, and if } \Delta_{k}^{m} \leq \frac{1}{4}, \\
\Delta_{k}^{m}, & \text { otherwise. }\end{cases}
\end{aligned}
$$




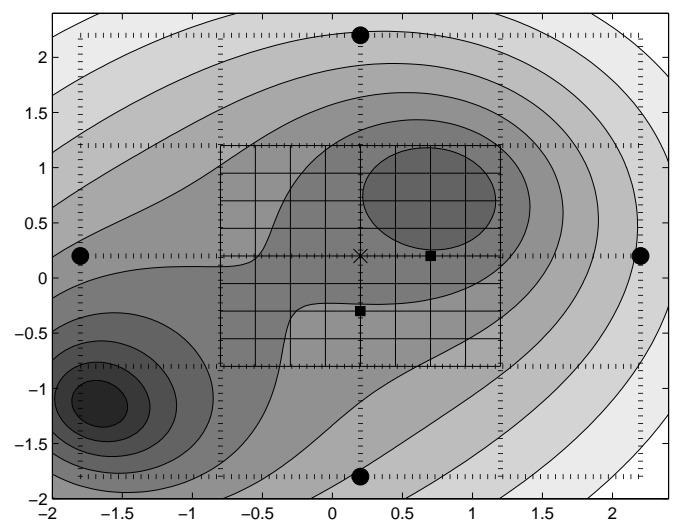

(a)

$\times \quad x_{k}$

- trial points in iteration $k+1$

- trial points in iteration $k+2$

$\triangle$ trial points in iteration $k+3$

- points in $S_{k}$

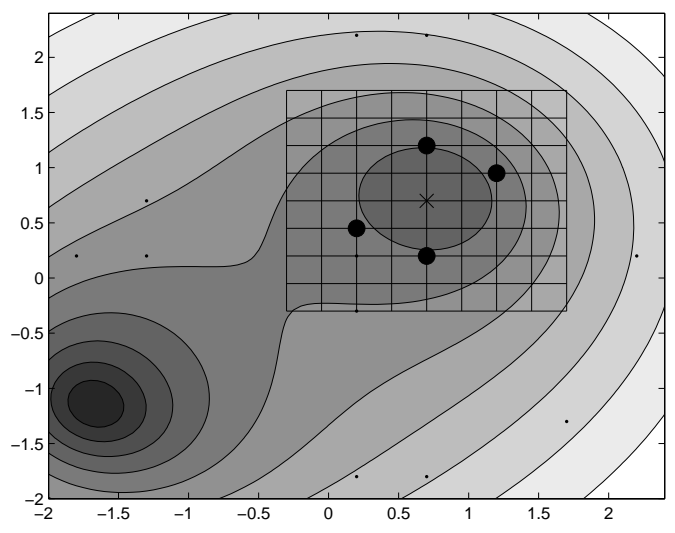

(b)

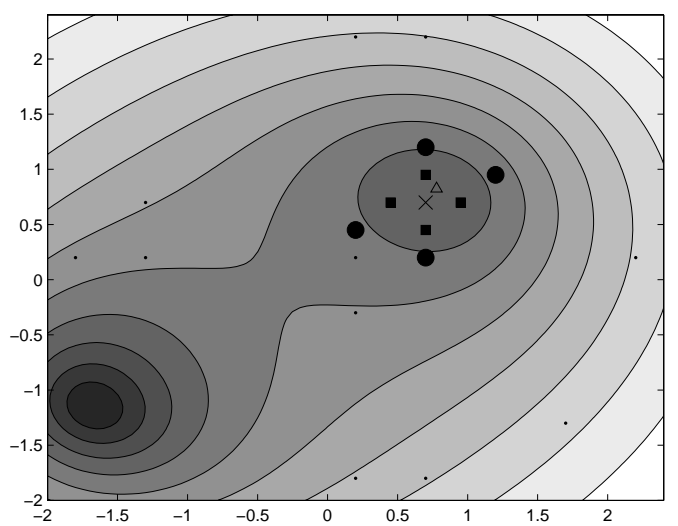

(c)

Figure 3. These three graphs show how a MADS algorithm can refine the mesh, choose different poll directions, and contract the search area. In Figure 3(a), the intersections of the dotted lines indicate points that met the criteria for frame points in the first iteration. From these possibilities, the algorithm chose the four points indicated by the large circles; these four points constitute $P_{1}$. At all of these points, the function is higher than at $x_{0}$, so the algorithm refined the mesh by setting $\Delta_{2}^{m}=\Delta_{1}^{m} / 4$. The intersections of the solid lines in the same graph indicate possible frame points for the second iteration. The algorithm evaluated the function at only two points in $P_{2}$ because the function is lower at the second point than at $x_{0}$. Figure $3(\mathrm{~b})$ shows how the mesh allows a MADS algorithm to choose different poll directions at each iteration. Figure 3(c) highlights three consecutive iterations of the algorithm. In the first two iterations, the algorithm is unable to find an improved mesh point, therefore it restricts the search area to be closer to $x_{k}$. This can be seen by looking at the points evaluated in the three iterations; the circles are the furthest away from $x_{k}$, the squares are closer, and the triangle is the closest. There is only one triangle because the function value at that point is lower than $f\left(x_{k}\right)$, so the algorithm stopped the POLL step and went on to the next iteration.

The poll size parameter $\Delta^{p}$ is updated according to the rule $\Delta_{k}^{p}=\sqrt{\Delta_{k}^{m}}$.

The example function in Figure 3 has two local minima in the chosen domain. The basin of 
attraction for the global minimum point is smaller than that of the other local minimum point. For the example problem, $\Omega=\left\{\left(x_{1}, x_{2}\right): x_{1} \in(-2,2.4), x_{2} \in(-2,2.4)\right\}$.

\section{Results}

All computation took place on System X, a cluster of 1100 dual-processor Mac G5 nodes.

NOMAD [4] is a $\mathrm{C}++$ implementation of the MADS class of algorithms. To take advantage of System X, NOMAD's implementation of the POLL step was parallelized using a master/worker paradigm. The master ran the MADS algorithm as presented in Section 4 and sent requests to the workers whenever objective function values were needed. NOMAD, started from the modeler's best point $s$, evaluated the objective function 135,000 times over 813 iterations using 128 processors, converging at a point for which the objective function value was 299 .

pVTDirect [7] is a parallel implementation of DIRECT written in Fortran 95. While the DIRECT algorithm does not have a traditional "starting point", the first sample in each subdomain is always taken at the center of the subdomain bounding box. For this problem, the bounding box was designed so that the modeler's best point [5] would be at the center and therefore would be evaluated before any other points. pVTDirect (with only one subdomain and $\epsilon=0$ ) ran for 473 iterations using 1024 processors and evaluated the objective function 1.5 million times, finding a point at which the objective function value was 212 .

The first set of runs (the results of which are illustrated in Figures 4 and 5) use the same initial conditions simulating all of the points, not different initial conditions as described in Section 2.2. (These runs led to the discovery of the necessity of the procedure in 2.2.) Figure 4 shows the progress that each algorithm was able to make in minimizing the objective function. While NOMAD was able to quickly find a better point than the modeler's best point, pVTDirect eventually found an even lower point. In a later run, NOMAD was started from pVTDirect's lowest point, but NOMAD was unable to make any further progress. After looking at Figure 4, it is tempting to believe that pVTDirect could have been stopped earlier (for instance, after 200,000 evaluations), and NOMAD started at pVTDirect's last best point could have found a point at which the objective function value was 212 or less. To test this, NOMAD was started at the best point at the 54th, 157th, and 239th iterations of pVTDirect. These points correspond to the beginning, middle, and end of the second lowest plateau in Figure 4. As shown in Figure 5, NOMAD started from the middle point converged to a point at which the objective function value was 210 . However, the NOMAD runs started at the beginning and end plateau points converge to worse 


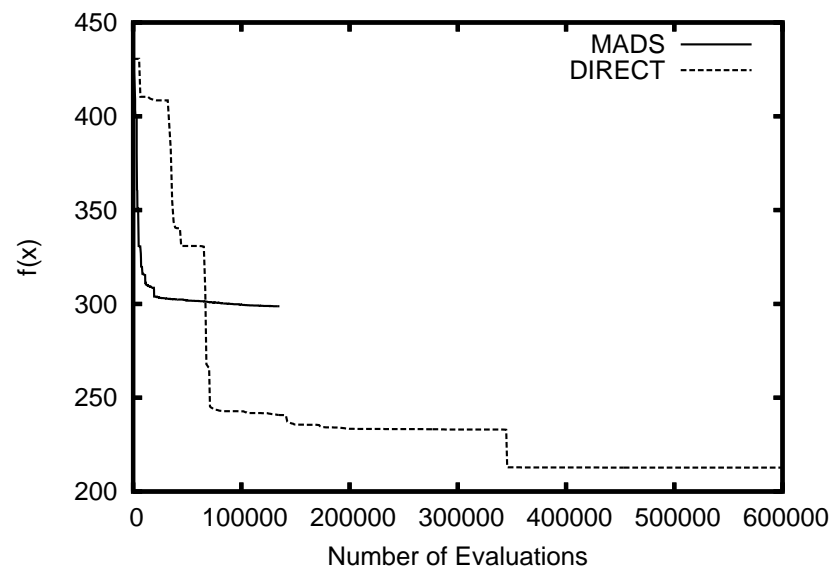

Figure 4. The objective function value at the best point found versus the number of evaluations for MADS and DIRECT. (The computations on which Figures 4 and 5 are based used a standard set of initial conditions for every simulation, not the more accurate updating of initial conditions described in Section 2.2.)

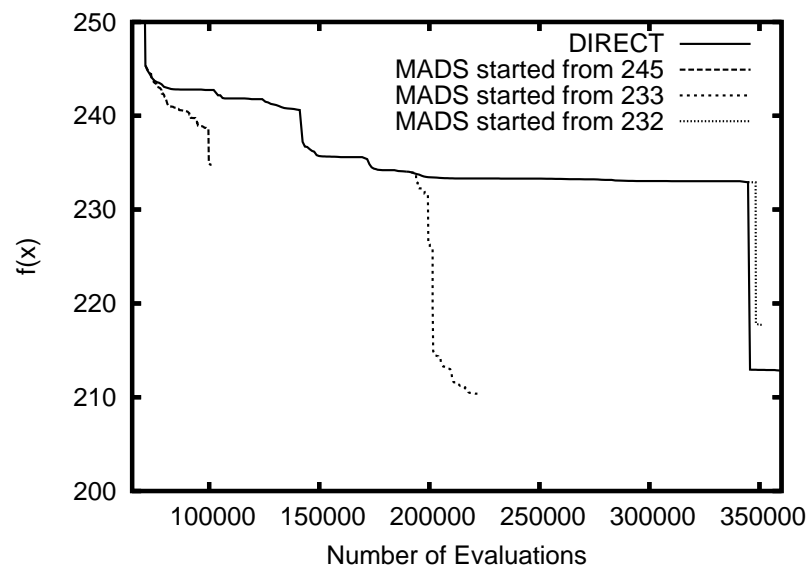

Figure 5. The performance of NOMAD when started from the best point at pVTDirect's 54 th, 157th, and 239th iterations. The plots are shown as if the NOMAD runs started as soon as the respective pVTDirect iterations completed.

points than pVTDirect's best point. These four extra NOMAD runs (including the one starting from pVTDirect's best point) show that using NOMAD to get more value out of a pVTDirect run is not simple.

When the initial conditions were generated (as described in Section 2.2) for the best points from the previously described runs, the points received considerably worse objective function values, leading to the realization that the initial conditions have to be chosen per Section 2.2 for each parameter vector. To remedy this, the objective function was modified to generate proper initial conditions (see Section 2.2) for each point before evaluating the point. NOMAD and pVTDirect were then rerun on the corrected objective function. For this set of runs, pVTDirect was run twice, 


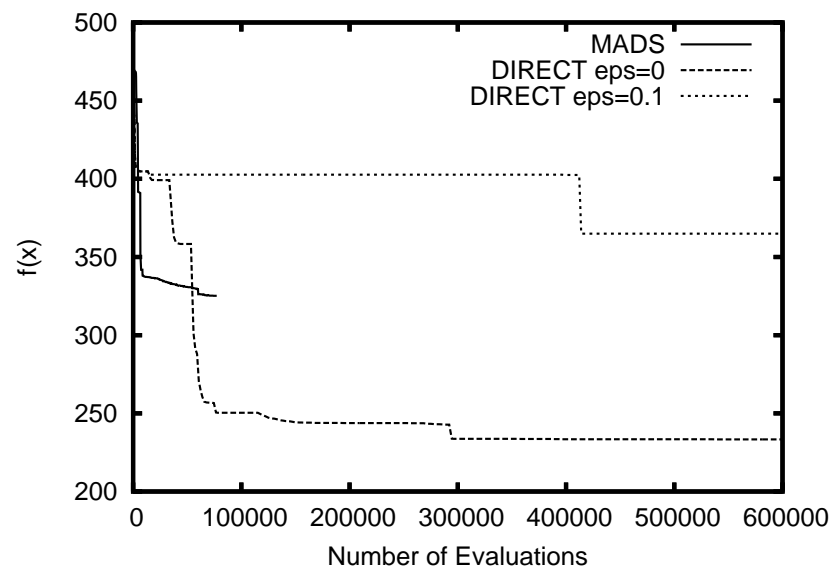

Figure 6. The objective function value at the best point found so far versus the number of evaluations for MADS, DIRECT with $\epsilon=0$, and DIRECT with $\epsilon=0.1$.

once with $\epsilon=0$ and again with $\epsilon=0.1$. Table 2 gives basic information for each method, and the results of these three runs are shown in Figure 6.

\begin{tabular}{lrrrr} 
Run & lowest $f$ & \# evaluations & \# CPUs & CPU hours \\
\hline MADS & 325 & 77,221 & 64 & 384 \\
DIRECT, $\epsilon=0$ & 233 & $1,243,429$ & 400 & 8,895 \\
DIRECT,$\epsilon=0.1$ & 365 & $1,452,597$ & 400 & 10,703
\end{tabular}

Table 2. Results for the runs that started from the best known point [5].

Figures 7, 8, and 9 give an idea of what areas of parameter space the different runs explored. These figures confirm that DIRECT evaluates points further away from the starting point than MADS. What is not shown in Figures 8 and 9 is that DIRECT found only 77,752 points that evaluated to less than 480 when $\epsilon$ was set to 0.1 , but it found 565,982 such points when $\epsilon$ was set to 0 , even though both runs evaluated approximately the same number of points. This, combined with the information shown in Figures 8 and 9, shows that setting $\epsilon$ to 0.1 caused DIRECT to spend more time dividing large boxes and less time refining small boxes.

However, Figure 5 showed that MADS may be able to quickly improve on the best points that DIRECT has found. Unfortunately, it is difficult to guess which points from DIRECT will be good starting points for MADS. To find points that scored well but were reasonably far apart, the following algorithm was used.

Step 1. Let $P$ be all of the points that DIRECT evaluated when $\epsilon$ was set to 0 . Set $k:=1$.

Step 2. Find $s_{k} \in P$ such that $\left\|s_{k}-s_{i}\right\|>2, i=1, \ldots, k-1$ and $f\left(s_{k}\right) \leq f(p)$ for all $p \in P$.

Step 3. If $f\left(s_{k}\right) \geq 300$, then stop, else set $k:=k+1$ and go to Step 2 . 


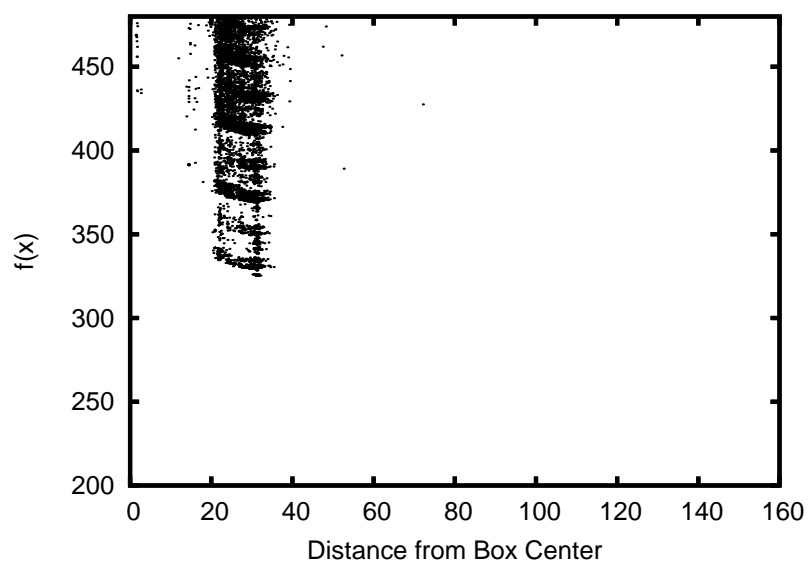

Figure 7. The distribution of points that evaluated to less than 480 when MADS ran.

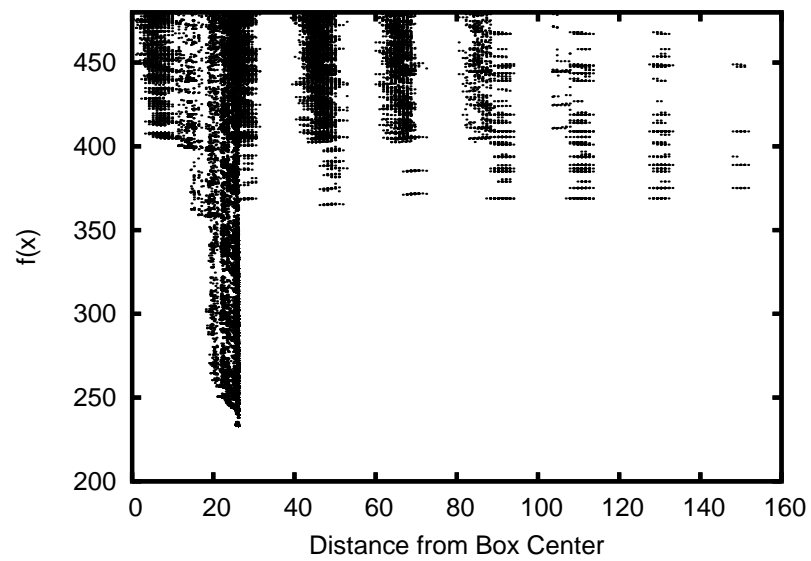

Figure 8. The distribution of points that evaluated to less than 480 when DIRECT ran with $\epsilon=0$.

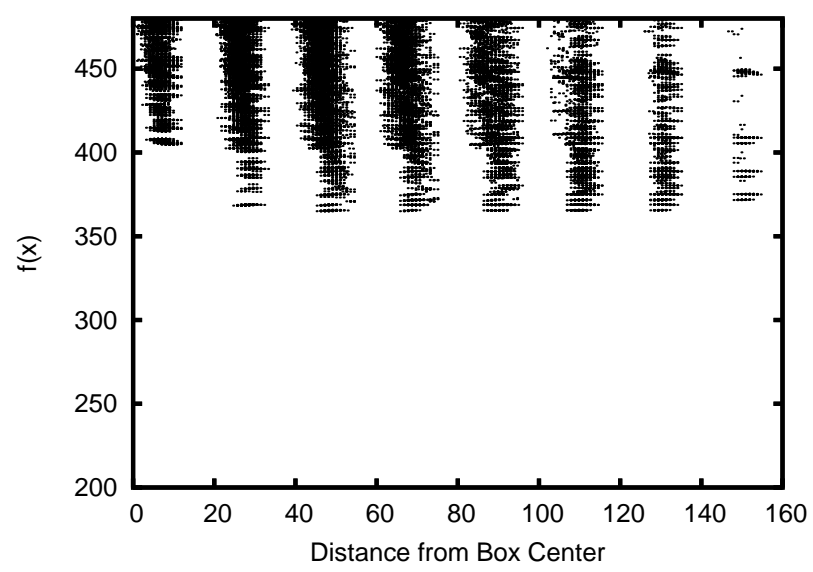

Figure 9. The distribution of points that evaluated to less than 480 when DIRECT ran with $\epsilon=0.1$. 
This algorithm yielded 13 points to use as starting points for MADS runs. The results of those runs are given in Table 3. In this table, there does not seem to be any relationship between $f\left(s_{i}\right)$ and the objective function value at the point to which MADS converges. It is also interesting that for 12 of the runs, MADS converges to a point that evaluates to 220-240, but the third run converges to a point that evaluates to a much lower score of 189 . This is the lowest score found by any of the runs.

\begin{tabular}{rrrrr}
$i$ & $f\left(s_{i}\right)$ & final $f$ & \# evaluations & CPU hours \\
\hline 1 & 233 & 233 & 1,590 & 12 \\
2 & 244 & 234 & 22,153 & 105 \\
3 & 244 & 189 & 76,978 & 384 \\
4 & 249 & 226 & 39,990 & 189 \\
5 & 250 & 230 & 82,551 & 384 \\
6 & 250 & 231 & 79,869 & 385 \\
7 & 255 & 224 & 59,884 & 266 \\
8 & 257 & 220 & 80,361 & 385 \\
9 & 260 & 228 & 86,251 & 384 \\
10 & 262 & 233 & 81,431 & 385 \\
11 & 291 & 228 & 79,110 & 384 \\
12 & 292 & 244 & 53,257 & 238 \\
13 & 293 & 238 & 84,035 & 384
\end{tabular}

Table 3. The MADS runs that started from points found during the DIRECT (with $\epsilon=0$ ) run. All MADS runs used 64 processors.

\section{Conclusion}

Even with a discontinuous objective function and a 143-dimension search domain, both DIRECT and MADS performed well. When $\epsilon$ was set to zero, DIRECT explored the parameter space and refined the boxes near local minima. When $\epsilon$ was set to 0.1, DIRECT used most of its evaluations to explore the parameter space. Any inferences about the choice of $\epsilon$ must be made with caution, since the box center here was already a very good point, and sizeable volumes of the 143-dimensional space still remain unsampled. MADS was almost always able to find a better point than its starting point, and it did so with far fewer evaluations than DIRECT. Using the two algorithms together yielded the lowest-scoring point.

The best previously known parameter vector from Chen et al. [5] has an objective function value of 470, and correctly models all of the mutants except (numbers from Appendix A) 7, 16, 35, 41, 45, 53, 76, 93, 97, 103, 104, and 110. The best point from DIRECT/MADS has an objective function value of 189 , and correctly models all but mutants 35, 41, 45, 53, 76, 93, and 110 . 


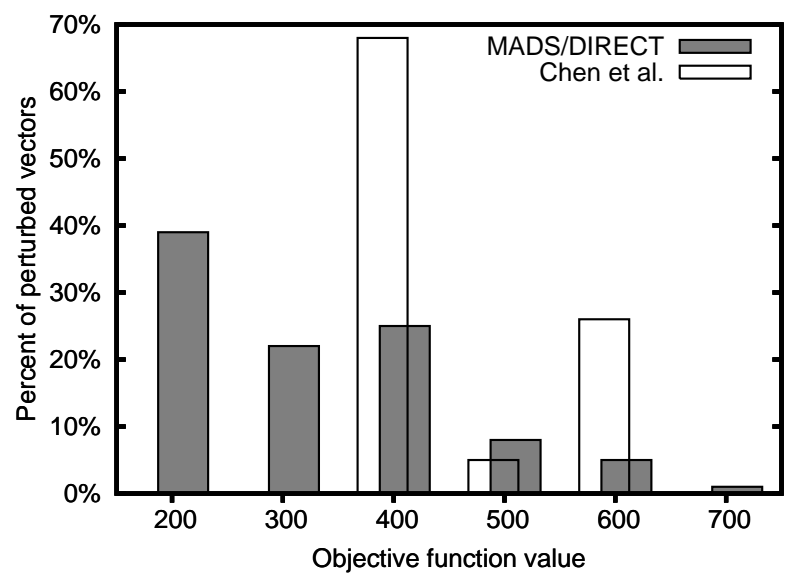

Figure 10. The best DIRECT/MADS parameter vector and the parameter vector from Chen et al. [5] were uniformly randomly perturbed by up to $\pm 0.5 \%$ in all dimensions, and the objective function was evaluated at each of the perturbed vectors. This figure shows the percentage of vectors that scored in $[x, x+100)$, for $x=200,300, \ldots, 700$.

How sensitive is the biological model (as reflected in the objective function) to local disturbances of these parameter vectors? If the DIRECT/MADS parameter values were rounded to two significant figures, would the model be such a good fit to the data? To investigate this question, random perturbations (up to $\pm 0.5 \%$ ) were applied to all of the parameter values in both the Chen et al. parameter vector and the best DIRECT/MADS parameter vector, and histograms of the resulting objective function values were computed (Figure 10). For the Chen et al. parameter vector, about $25 \%$ of perturbations (white bars in Figure 10) give significantly worse fits to the data, a reflection of the fact that behavior of the model is quite sensitive to a small number of the parameters, as described in detail in [5]. The DIRECT/MADS parameter vector (grey bars in Figure 10) appears to be not only better but also its perturbations are less likely to produce inferior models than for the Chen et al. parameter vector. Thus the combination of DIRECT and MADS to perform parameter optimization on discontinuous objective functions in very high dimensional (> 100) parameter spaces is not only feasible computationally but also can find "optimal" parameter vectors that improve on the best estimates of expert modelers.

\section{Acknowledgments}

This work was partly supported by Defense Advanced Research Projects Agency (DARPA) grant F30602-02-0572. 


\section{References}

1. Allen, N. A., Calzone, L., Chen, K. C., Ciliberto, A., Ramakrishnan, N., Shaffer, C. A., Sible, J. C., Tyson, J. J., Vass, M. T., Watson, L. T., and Zwolak, J. W. (2003), Modeling regulatory networks at Virginia Tech, OMICS 7: 285-299.

2. Allen, N. A., Chen, K. C., Tyson, J. J., Shaffer, C. A., and Watson, L. T, Computer evaluation of network dynamics models with application to cell cycle control in budding yeast, IEE Systems Biology (to appear).

3. Allen, N. A., Shaffer, C. A., Ramakrishnan, N., Vass, M. T., and Watson L. T. (2003), Improving the development process for eukaryotic cell cycle models with a modeling support environment, Simulation 79: 674-688.

4. Audet, C. and Dennis Jr., J. E., Mesh adaptive direct search algorithms for constrained optimization, SIAM Journal on Optimization (to appear).

5. Chen, K. C., Calzone, L., Csikasz-Nagy, A., Cross, F. R., Novak, B., and Tyson, J. J. (2004), Integrative analysis of cell cycle control in budding yeast, Molecular Biology of the Cell, 15: 3841-3862.

6. Coope, I. D. and Price, C. J. (2000), Frame based methods for unconstrained optimization, Journal of Optimization Theory and Applications 107: 261-274.

7. He, J., Sosonkina, M., Shaffer, C. A., Tyson, J. J., Watson, L. T., and Zwolak, J. W. (2004), A hierarchical parallel scheme for a global search algorithm, in Proc. High Performance Computing Symposium 2004, J. Meyer (ed.), Soc. for Modeling and Simulation Internat., San Diego, CA, 43-50.

8. He, J., Sosonkina, M., Shaffer, C. A., Tyson, J. J., Watson, L. T., and Zwolak, J. W. (2004), A hierarchical parallel scheme for global parameter estimation in systems biology, in Proceedings of the 18th International Parallel \& Distributed Processing Symposium, CD-ROM, IEEE Computer Soc., Los Alamitos, CA, 9 pages.

9. He, J., Sosonkina, M., Watson, L. T., Verstak, A., and Zwolak, J. W. (2005), Data-distributed parallelism with dynamic task allocation for a global search algorithm, in Proc. High Performance Computing Symposium 2005, M. Parashar and L. Watson (eds.), Society for Modeling and Simulation International, San Diego, CA, 164-172.

10. He, J., Watson, L. T., Ramakrishnan, N., Shaffer, C. A., Verstak, A., Jiang, J., Bae, K., and Tranter, W. H. (2002), Dynamic data structures for a direct search algorithm, Computational Optimization and Applications 23: 5-25.

11. Jones, D. R., Perttunen, C. D., and Stuckman, B. E. (1993), Lipschitzian optimization without the Lipschitz constant, Journal of Optimization Theory and Applications, 79: 157-181.

13. Murray, A. and Hunt, T. (1993), The Cell Cycle: an Introduction, Oxford University Press, New York, 1993.

14. Nasmyth, K. (1996) At the heart of the budding yeast cell cycle, Trends in Genetics, 12: 405-412.

15. Nurse, P. (2000), A long twentieth century of the cell cycle and beyond, Cell, 100: 71-78. 


\section{Appendix A: Mutants}

Listed below are the phenotypic characteristics of all the mutants used to construct the objective function. The data are expressed as a six-tuple $(v, g, m, a, t, c)$ as described in Section 3. The meanings of the values for $t$ are described in [2]. For all fields other than viability, a dash $(-)$ means that the data is either not available or not applicable for that mutant.

Mutant name

1. Wild type in glucose

2. Wild type in galactose

3. $\operatorname{cln} 1 \Delta \operatorname{cln} 2 \Delta$

4. $G A L-C L N 2 \operatorname{cln} 1 \Delta \operatorname{cln} 2 \Delta$

5. $\operatorname{cln} 1 \Delta \operatorname{cln} 2 \Delta \operatorname{sic} 1 \Delta$

6. $\operatorname{cln} 1 \Delta \operatorname{cln} 2 \Delta c \operatorname{dh} 1 \Delta$

7. GAL-CLN2 $\operatorname{cln} 1 \Delta \operatorname{cln} 2 \Delta c d h 1 \Delta$

8. $\quad \ln 3 \Delta$

9. GAL-CLN3

10. $b c k 2 \Delta$

11. Multi-copy BCK2

12. $\operatorname{cln} 1 \Delta c \ln 2 \Delta$ bck $2 \Delta$

13. $\operatorname{cln} 3 \Delta b c k 2 \Delta$

14. $\operatorname{cln} 3 \Delta$ bck $2 \Delta$ GAL-CLN2 $\operatorname{cln} 1 \Delta \operatorname{cln} 2 \Delta$

15. $\operatorname{cln} 3 \Delta$ bck $2 \Delta$ multi-copy CLN2

16. $\operatorname{cln} 3 \Delta$ bck $2 \Delta$ GAL-CLB5

17. $\operatorname{cln} 3 \Delta$ bck $2 \Delta \operatorname{sic} 1 \Delta$

18. $\operatorname{cln} 1 \Delta c \ln 2 \Delta \operatorname{cln} 3 \Delta$

19. $c \ln 1 \Delta c \ln 2 \Delta c \ln 3 \Delta G A L-C L N 2$

20. $\operatorname{cln} 1 \Delta \operatorname{cln} 2 \Delta \operatorname{cln} 3 \Delta$ GAL-CLN3

21. $\operatorname{cln} 1 \Delta \operatorname{cln} 2 \Delta \operatorname{cln} 3 \Delta \operatorname{sic} 1 \Delta$

22. $\operatorname{cln} 1 \Delta c \ln 2 \Delta \operatorname{cln} 3 \Delta c d h 1 \Delta$

23. $\operatorname{cln} 1 \Delta \operatorname{cln} 2 \Delta \operatorname{cln} 3 \Delta$ multi-copy CLB5

24. $\operatorname{cln} 1 \Delta \operatorname{cln} 2 \Delta \operatorname{cln} 3 \Delta$ GAL-CLB5

25. $\quad \ln 1 \Delta c \ln 2 \Delta \operatorname{cln} 3 \Delta$ multi-copy $B C K 2$

26. $\operatorname{cln} 1 \Delta \operatorname{cln} 2 \Delta \operatorname{cln} 3 \Delta$ GAL-CLB2

27. $\operatorname{cln} 1 \Delta c \ln 2 \Delta \operatorname{cln} 3 \Delta$ apc-ts

28. $\operatorname{sic} 1 \Delta$

29. GAL-SIC1

30. GAL-SIC1-dbD

31. GAL-SIC1 $\operatorname{cln} 1 \Delta \operatorname{cln} 2 \Delta$

32. GAL-SIC1 $\operatorname{cln} 1 \Delta \operatorname{cln} 2 \Delta c d h 1 \Delta$

33. GAL-SIC1 GAL-CLN2 $\operatorname{cln} 1 \Delta c \ln 2 \Delta$

34. GAL-SIC1 GAL-CLN2 $\operatorname{cln} 1 \Delta \operatorname{cln} 2 \Delta c d h 1 \Delta$

35. $\operatorname{sic} 1 \Delta \operatorname{cdh} 1 \Delta$

36. $\operatorname{sic} 1 \Delta \operatorname{cdh} 1 \Delta$ GALL-CDC20

37. $c d h 1 \Delta$

38. Cdh1 constitutively active

39. $c d c 6 \Delta 2-49$

40. $\operatorname{sic} 1 \Delta \operatorname{cdc} 6 \Delta 2-49$

41. $\operatorname{cdh} 1 \Delta \operatorname{cdc} 6 \Delta 2-49$

42. $\operatorname{clb} 1 \Delta$ clb2 $\Delta$

43. GAL-CLB2

$\begin{array}{lrrccc}v & g & m & a & t & c \\ \text { viable } & 35.2 & 1 & - & - & - \\ \text { viable } & 109 & 1 & - & - & - \\ \text { viable } & - & 2 & - & - & - \\ \text { viable } & - & 0.5 & - & - & - \\ \text { viable } & - & - & - & - & - \\ \text { viable } & - & - & - & - & - \\ \text { viable } & - & 1.7 & - & - & - \\ \text { viable } & - & 1.7 & - & - & - \\ \text { viable } & - & 0.44 & - & - & - \\ \text { viable } & - & 1.4 & - & - & - \\ \text { viable } & - & 0.8 & - & - & - \\ \text { viable } & - & 1.7 & - & - & - \\ \text { inviable } & - & - & \text { licensed } & 5 & 0 \\ \text { viable } & - & - & - & - & - \\ \text { inviable } & - & - & \text { licensed } & 5 & - \\ \text { inviable } & - & - & - & - & - \\ \text { inviable } & - & - & - & - & - \\ \text { inviable } & - & - & \text { licensed } & 5 & 0 \\ \text { viable } & - & - & - & - & - \\ \text { viable } & - & - & - & - & - \\ \text { viable } & 10 & 3.5 & - & - & - \\ \text { inviable } & - & - & \text { separated } & 3 & - \\ \text { viable } & - & - & - & - & - \\ \text { viable } & - & - & - & - & - \\ \text { viable } & - & - & - & - & - \\ \text { inviable } & - & - & \text { licensed } & 5 & 0 \\ \text { inviable } & - & - & \text { aligned } & 3 & 0 \\ \text { viable } & 15 & 1 & - & - & - \\ \text { viable } & 135 & 2 & - & - & - \\ \text { inviable } & - & - & \text { licensed } & 5 & 0 \\ \text { inviable } & - & - & \text { licensed } & 5 & - \\ \text { inviable } & - & - & \text { licensed } & 5 & - \\ \text { viable } & - & - & - & - & - \\ \text { viable } & - & - & - & - & - \\ \text { inviable } & - & - & \text { unlicensed } & 1 & 1 \\ \text { viable } & - & - & - & - & - \\ \text { viable } & - & 0.6 & - & - & - \\ \text { inviable } & - & - & \text { fired } & 3 & - \\ \text { viable } & - & - & - & - & - \\ \text { viable } & - & - & - & - & - \\ \text { viable } & 20 & 2.0 & - & - & - \\ \text { inviable } & - & - & \text { fired } & 3 & 0 \\ \text { viable } & - & - & - & - & - \\ \text { viable } & & & & & \end{array}$


44. Multicopy GAL-CLB2

45. GAL-CLB2 sic1 $\triangle$

46. GAL-CLB2 cdh1 $\triangle$

47. $C L B 2-d b \Delta$

48. $C L B 2-d b \Delta$ in galactose

49. CLB2-db $\Delta$ multicopy SIC1

50. CLB2-dbD GAL-SIC1

51. CLB2-db $\Delta$ clb5 $\Delta$

52. CLB2-db $\Delta$ clb5 $\Delta$ in galactose

53. GAL-CLB2-db $\Delta$

54. $\operatorname{clb5} \Delta$ clb6 $\Delta$

55. $\operatorname{cln} 1 \Delta \operatorname{cln} 2 \Delta \operatorname{clb5} \Delta \operatorname{clb} 6 \Delta$

56. GAL-CLB5

57. GAL-CLB5 sic1 $\triangle$

58. GAL-CLB5 cdh1 $\triangle$

59. CLB5-dbD

60. $C L B 5-d b \Delta \operatorname{sic} 1 \Delta$

61. $C L B 5-d b \Delta$ pds1 $\Delta$

62. CLB5-dbs pds $1 \Delta c d c 20 \Delta$

63. GAL-CLB5-db $\Delta$

64. cdc20-ts

65. $c d c 20 \Delta$ clb5 $\Delta$

66. $c d c 20 \Delta p d s 1 \Delta$

67. $c d c 20 \Delta$ pds $1 \Delta$ clb5 $\Delta$

68. GAL-CDC20

69. $c d c 20$-ts $\operatorname{mad} 2 \Delta$

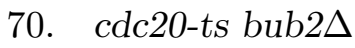

71. $p d s 1 \Delta$

72. esp1-ts

73. PDS1-db $\Delta$

74. GAL-PDS1-db $\Delta$

75. GAL-PDS1-dbD esp1-ts

76. GAL-ESP1 cdc20-ts

77. tem1 $\triangle$

78. GAL-TEM1

79. tem1-ts GAL-CDC15

80. tem1 1 net1-ts

81. tem1-ts multicopy CDC14

82. $c d c 15 \Delta$

83. Multicopy CDC15

84. cdc15-ts multicopy TEM1

85. cdc15 $\Delta$ net1-ts

86. cdc15-ts multicopy CDC14

87. net1-ts

88. GAL-NET1

89. cdc14-ts

90. GAL-CDC14

91. GAL-NET1 GAL-CDC14

92. net1 $\Delta$ cdc20-ts

93. cdc14-ts GAL-SIC1

$\begin{array}{lccccc}\text { inviable } & - & - & \text { separated } & 3 & 0 \\ \text { inviable } & - & - & \text { separated } & 3 & 0 \\ \text { inviable } & - & - & - & - & - \\ \text { inviable } & - & - & \text { separated } & 3 & - \\ \text { inviable } & - & - & \text { separated } & 3 & - \\ \text { viable } & - & - & - & - & - \\ \text { viable } & - & - & - & - & - \\ \text { inviable } & - & - & \text { separated } & 3 & 0 \\ \text { viable } & - & - & - & - & - \\ \text { inviable } & - & - & \text { separated } & 3 & - \\ \text { viable } & 65 & - & - & - & - \\ \text { inviable } & - & - & \text { licensed } & 5 & 0 \\ \text { viable } & - & - & - & - & - \\ \text { inviable } & - & - & \text { unlicensed } & - & 1 \\ \text { inviable } & - & - & - & - & - \\ \text { viable } & - & - & - & - & - \\ \text { inviable } & - & - & - & - & 1 \\ \text { viable } & - & - & - & - & - \\ \text { inviable } & - & - & \text { separated } & 3 & 0 \\ \text { inviable } & - & - & - & - & 1 \\ \text { inviable } & - & - & \text { aligned } & 3 & 0 \\ \text { inviable } & - & - & \text { aligned } & 3 & 0 \\ \text { inviable } & - & - & \text { separated } & 3 & 0 \\ \text { viable } & - & - & - & - & - \\ \text { inviable } & - & - & \text { fired } & 10 & 0 \\ \text { inviable } & - & - & \text { aligned } & 3 & 0 \\ \text { inviable } & - & - & \text { aligned } & 3 & 0 \\ \text { viable } & - & - & - & - & - \\ \text { inviable } & - & - & \text { aligned } & 1 & 0 \\ \text { inviable } & - & - & \text { aligned } & 1 & 0 \\ \text { inviable } & - & - & \text { aligned } & 1 & 0 \\ \text { inviable } & - & - & \text { aligned } & 1 & 0 \\ \text { inviable } & - & - & \text { separated } & 3 & 0 \\ \text { inviable } & - & - & \text { separated } & 3 & 0 \\ \text { viable } & - & - & - & - & - \\ \text { viable } & - & - & - & - & - \\ \text { viable } & - & - & - & - & - \\ \text { viable } & - & - & - & - & - \\ \text { inviable } & - & - & \text { separated } & 3 & 0 \\ \text { viable } & - & - & - & - & - \\ \text { inviable } & - & - & - & - & - \\ \text { viable } & - & - & - & - & - \\ \text { viable } & - & - & - & - & - \\ \text { viable } & 50 & - & - & - & - \\ \text { inviable } & - & - & - & - & - \\ \text { viable } & - & - & - & - & - \\ & - & - & \text { separated } & 3 & 0 \\ \text { inviable } & - & - & \text { separated } & 3 & 0 \\ \text { iniable } & - & - & \text { licensed } & 5 & 0 \\ \text { inale } & - & - & \text { aligned } & 1 & - \\ \text { inale } & & & & & \end{array}$


94. TAB6-1

95. TAB6-1 cdc15

96. TAB6-1 clb5 $\mathrm{clb6 \Delta}$

97. TAB6-1 CLB1 clb2A

98. $\operatorname{mad} 2 \Delta$

99. bub2A

100. $\operatorname{mad} 2 \Delta$ bub2 $\Delta$

101. $A P C-A$

102. $A P C-A \operatorname{cdh} 1 \Delta$

103. $A P C-A c d h 1 \Delta$ in galactose

104. $A P C-A$ cdh $1 \Delta$ multicopy SIC1

105. APC-A cdh1 $\triangle$ GAL-SIC1

106. APC-A cdh1 $\triangle$ multicopy $C D C 6$

107. APC-A cdh1 $\triangle$ GAL-CDC6

108. APC-A cdh1 $\Delta$ multicopy $C D C 20$

109. Swi5

110. $\operatorname{sic} 1 \Delta \operatorname{cdc} 6 \Delta 2-49 \operatorname{cdh} 1 \Delta$

111. sic1 $\Delta$ cdc6 $\Delta 2-49$ cdh1 $\operatorname{GALL}-C D C 20$

112. $A P C-A$ cdh $1 \Delta$ clb5 $\Delta$

113. APC-A cdh1 $\Delta$ pds1 $\Delta$

114. $A P C-A$ sic1 $\triangle$

115. APC-A GAL-CLB2

$\begin{array}{lrrccc}\text { viable } & - & - & - & - & - \\ \text { viable } & - & - & - & - & - \\ \text { inviable } & - & - & \text { licensed } & 5 & 0 \\ \text { viable } & - & - & - & - & - \\ \text { viable } & 35 & 1 & - & - & - \\ \text { viable } & 35 & 1 & - & - & - \\ \text { viable } & - & - & - & - & - \\ \text { viable } & 20 & 1.5 & - & - & - \\ \text { inviable } & - & - & \text { separated } & 3 & - \\ \text { viable } & - & - & - & - & - \\ \text { viable } & - & - & - & - & - \\ \text { viable } & - & - & - & - & - \\ \text { viable } & - & - & - & - & - \\ \text { viable } & - & - & - & - & - \\ \text { viable } & - & - & - & - & - \\ \text { viable } & 20 & - & - & - & - \\ \text { inviable } & - & - & \text { fired } & 3 & 1 \\ \text { viable } & - & - & - & - & - \\ \text { inviable } & - & - & - & - & - \\ \text { inviable } & - & - & - & - & - \\ \text { viable } & - & - & - & - & - \\ \text { inviable } & - & - & \text { separated } & 3 & -\end{array}$


Figure 1. The five stages of the cell cycle, delineated by the events described in the first rule of viability. The four biological phases of the cell are above the stages, and two of the phases within M phase are shown below their corresponding stages.

Figure 2. These graphs show the function evaluations that DIRECT performed after zero, one, five, and eleven iterations. Comparing the first and second graphs shows how DIRECT divides a two-dimensional box. The second and third graphs include the rectangles that DIRECT had created. After five iterations, DIRECT has found the global minimum at $(-1.8,-1.1)$. After the fifth iteration DIRECT has explored the domain, subdividing most of the larger boxes. After eleven iterations, DIRECT has evaluated the function at points near the local minimum.

Figure 3. These three graphs show how a MADS algorithm can refine the mesh, choose different poll directions, and contract the search area. In Figure 3(a), the intersections of the dotted lines indicate points that met the criteria for frame points in the first iteration. From these possibilities, the algorithm chose the four points indicated by the large circles; these four points constitute $P_{1}$. At all of these points, the function is higher than at $x_{0}$, so the algorithm refined the mesh by setting $\Delta_{2}^{m}=\Delta_{1}^{m} / 4$. The intersections of the solid lines in the same graph indicate possible frame points for the second iteration. The algorithm evaluated the function at only two points in $P_{2}$ because the function is lower at the second point than at $x_{0}$. Figure 3(b) shows how the mesh allows a MADS algorithm to choose different poll directions at each iteration. Figure 3(c) highlights three consecutive iterations of the algorithm. In the first two iterations, the algorithm is unable to find an improved mesh point, therefore it restricts the search area to be closer to $x_{k}$. This can be seen by looking at the points evaluated in the three iterations; the circles are the furthest away from $x_{k}$, the squares are closer, and the triangle is the closest. There is only one triangle because the function value at that point is lower than $f\left(x_{k}\right)$, so the algorithm stopped the POLL step and went on to the next iteration.

Figure 4. The objective function value at the best point found versus the number of evaluations for MADS and DIRECT. (The computations on which Figures 4 and 5 are based used a standard set of initial conditions for every simulation, not the more accurate updating of initial conditions described in Section 2.2.)

Figure 5. The performance of NOMAD when started from the best point at pVTDirect's 54th, 157 th, and 239th iterations. The plots are shown as if the NOMAD runs started as soon as the respective pVTDirect iterations completed.

Figure 6 . The objective function value at the best point found so far versus the number of evaluations for MADS, DIRECT with $\epsilon=0$, and DIRECT with $\epsilon=0.1$.

Figure 7. The distribution of points that evaluated to less than 480 when MADS ran.

Figure 8. The distribution of points that evaluated to less than 480 when DIRECT ran with $\epsilon=0$.

Figure 9. The distribution of points that evaluated to less than 480 when DIRECT ran with $\epsilon=0.1$.

Figure 10. The best DIRECT/MADS parameter vector and the parameter vector from Chen et al. [5] were randomly perturbed by up to $\pm 0.5 \%$ in all dimensions, and the objective function was evaluated at each of the perturbed vectors. This figure shows the percentage of vectors that scored in $[x, x+100)$, for $x=200,300, \ldots, 700$. 